\title{
Opinions and Attitudes of Health Care Staff towards Mobile Phone Messaging
}

\section{livari Bäck* and Kari Mäkelä}

Department of Biomedical Engineering, Tampere University of Technology, Finland

\begin{abstract}
Mobile phone text messaging (Short Message Service, SMS) has proved to be a powerful tool for many tasks in health care. However, this technology has not yet been deployed in the hospital environment in full scale. The goal of this study was to understand the attitudes, opinions and expectations of the hospital staff about the mobile phone messaging in health care in Seinäjoki Central Hospital, which is located in Western Finland. SMS based applications have been used in the hospital in psychiatric care and in various applications related to appointments. However, there are plans to extend the use of the mobile messaging tools on new areas in the hospital. For this reason, we needed the opinion data from the hospital personnel. We conducted a survey including structured questionnaire containing 19 questions on text messaging and multimedia messaging use in health care applications. The participants were allowed to give numerical opinion for each question, and also add free-text comments. We received 218 responses, and analyzed them numerically as well as based on the free-text feedback given by the participants. Although the survey results show good acceptance of the text messages as, for example, reminders of appointments, the participants expressed various concerns such as privacy matters and the risk of misunderstandings in the message content. The survey proved also to be a good way of sharing information about this new technology to be introduced in hospital environment.
\end{abstract}

\section{Introduction}

Mobile computing and communication technology interventions for improving health care and health service outcomes, referred as M-health [1] are used nowadays on a wide range from data collection and information transmission to supporting health behavior change. Short Message Service (SMS) and Multimedia Messaging Service (MMS) are the most widely used mobile communication methods after phone calls. In principle, text message can be used either as a one-way communication to provide the user information such as reminder, alert, or as a two way communication that enables the user to send and receive information (such as question and answer). Mobile messages are an excellent aid for communication when there is a need to submit information also at long distances or without well working health infrastructure, or when the people cannot physically meet the health care workers. Provided that the cost of the text message is very low and it is available to everyone practically, it has been found useful to employ the text messaging in many routine-like health care applications. Typical examples of these application areas are different kinds of reminders [2] and instructions, reporting of laboratory test results or home measurements, remote controlling and monitoring etc. By using this kind of communication method, it is possible to save resources by avoiding unnecessary hospital visits and phone calls, and make health care process more efficient by automatically sending, receiving, storing and processing the patient data. Currently there are some surveys and literature reviews available about the use of text messaging in certain areas of health care. The use of SMS reminders to improve attendance at hospital appointments has been reviewed by hasvold and wootton [2]. This review, containing 29 studies, suggests that the weighted mean relative change in non-attendance was $29 \%$, when automated reminders were used. In a very recent paper of Wei et al. [3], a literature review on the use of text messaging for clinical and healthy behavior interventions is presented. 16 of 24 reviewed articles were randomized controlled trials. Among these 16 trial articles, 10 reported significant improvements with interventions and six reported differences suggesting positive trends. Also cole-lewis and kershaw [4] presented a review on the behavior change interventions for disease management and prevention delivered through text messaging. The review identified 9 sufficiently powered studies, and 8 of them found evidence to support text messaging as a tool for behavior change. The state-of-the-art of the use of the mobile phones and text messaging interventions in improving health outcomes and processes of care are reviewed by krishna et al. [5]. The main finding of the review was that mobile phone reminder, disease monitoring and management and education can improve health outcomes and care processes.

\section{Mobile Phone Messaging in Health Care}

In our research project, one goal is to develop working mobile phone messaging systems for a hospital environment. As a preliminary step we carried out a background study of the existing mobile phone messaging applications in health care. The review was carried out as an internet database search in April-July 2011, and it was based on a search of pub med database with relevant keywords. The entire study can be found in [6]. Based on the review and related analysis, we identified the following categories of mobile phone messages in health care:

\section{Text messaging applications}

- Applications related to appointments

The text message applications are often used to remind or inform the patients about the appointments $[2,7,8]$.

- Alerts

Text messages can be used as a tool for sending urgent outbreak alerts and warnings to multiple persons in multiple locations

*Corresponding author: livari Bäck, Ph.D, Department of Biomedical Engineering Tampere University of Technology, Seinäjoki Unit, Koskenalantie 16, FI-60220 Seinäjoki, Finland; E-mail: iivari.back@uwasa.fi

Received September 13, 2012; Accepted September 22, 2012; Published Spetember 25, 2012

Citation: Bäck I, Mäkelä K (2012) Opinions and Attitudes of Health Care Staff towards Mobile Phone Messaging. Primary Health Care 2:125. doi:10.4172/2167. 1079.1000125

Copyright: (c) 2012 Bäck I, et al. This is an open-access article distributed under the terms of the Creative Commons Attribution License, which permits unrestricted use, distribution, and reproduction in any medium, provided the original author and source are credited. 
Citation: Bäck I, Mäkelä K (2012) Opinions and Attitudes of Health Care Staff towards Mobile Phone Messaging. Primary Health Care 2:125. doi:10.4172/2167-1079.1000125

Page 2 of 5

simultaneously [9]. Also warnings about invasive diseases [10] belong to this category.

- Information Services

Text messaging makes it possible to send health related information to the patients. The information can be, for example, the results of medical examinations [8], information about medication [11], education [12,13] etc.

- Two-way communication between the patients and hospital There are several existing applications in which the patients can send the results of home measurements to hospital server by using text message. The server automatically sends feedback information as returning message. Typical application fields of this kind of messaging can be found in diabetes management $[14,15]$, asthma management $[16,17]$. In an application related to psychiatry [18], the patients receive answers to their questions from the hospital staff.

\section{- Alarms}

The mobile phone carried by the patient can send an automatic message in case of a detected emergency. The typical use cases of this type of messaging include the monitoring of the patients that have some chronic disease [19] or activity monitoring in the homes of elderly [20].

\section{- Adherence}

Text messaging applications can be used to support selfmanagement of the patients suffering from some chronic diseases, or participants of a program aiming at lifestyle changes or therapeutic use. The messages may involve adhering to drug
"Do you find mobile phone text messages or multimedia messaging a feasible way of communication in the following scenarios?"

A) Applications related to appointments,

1 Text messages are used to remind the patients about appointments for doctor's consultation

2 Text messages are used to remind the patients about appointments for medical operations

Text messages are used to inform the patient about changed or canceled ap-

3 pointments. The patient can accept or cancel the proposed new appointment time by text message.

Text messages are used to inform the patient about a possibility to get an ap-

4 pointment time which has been canceled by some other patient. The patient can accept or cancel the proposed new appointment time by text message

\section{B) Alerts}

5 A text message warning about a disease or epidemic is automatically sent to a certain group of people

6 Text message is used to warn people who have been in contact with a person suffering from an invasive disease

\section{C) Information services}

7 The results of laboratory examinations are sent to the patient via text message

8 The patient is asked via text message to contact the hospital to make an ap-

pointment for additional examinations

\section{D)Two-way communication between patients and hospital staff,}

The patients have a possiblity to send the results of home measurements (like

9 blood pressure, glucose value or respiratory measurements) to hospital server by text message.

10 The patients participating to a certain treatment have a possibility to send information or questions related to the treatment to hospital via text message.

\section{E) Alarms}

Mobile phone carried by the patient can send an emergency message to hospi-

11 tal or other care giver in case of e.g. syncope or convulsion. The message may contain also the GPS coordinates of the patient.

F) Adherence

12 A text message reminder about medication is sent to the patient

13 Reminder message can contain additional medication information and advice

14 The patient has a possiblity to send returning message to report about prolems

with medication (e.g. missing doses or side effects)

15 People participating to healthy lifestyle programmes (e.g. Weight management

or smoking cessation) are sent advicing and encouraging messages Applications of multimedia messages.

16 Health care personnel has a possiblity to send pictures taken from the patient

16 via multimedia message to doctor/specialist for teleconsultation.

17 The patients have a possiblity to send pictures taken by themselves to hospital

17 (e.g. Surgery wounds, skin conditions etc)

18 The information sent to patient transmitted to an audio message (in case of

8 visually impaired patients)

19 The patients can be sent information as video messages

\begin{tabular}{|c|c|c|c|c|c|}
\hline \multicolumn{6}{|c|}{ All participants (218) } \\
\hline Yes (1) & Maybe (2) & Neutral (3) & $\begin{array}{l}\text { Maybe not } \\
\text { (4) }\end{array}$ & No (5) & $\begin{array}{c}\text { Average } \\
\text { value }\end{array}$ \\
\hline $61(27,9 \%)$ & $68(31,1 \%)$ & $28(12,8 \%)$ & $33(15,1 \%)$ & $29(13,2 \%)$ & 2.55 \\
\hline $125(57,9 \%)$ & $49(22.7 \%)$ & $12(5,6 \%)$ & $14(6,5 \%)$ & $16(7,4 \%)$ & 1.83 \\
\hline $133(59,9 \%)$ & $47(21,2 \%)$ & $8(3,6 \%)$ & $14(6,5 \%)$ & $20(9 \%)$ & 1.83 \\
\hline $130(59,1 \%)$ & $42(19,1 \%)$ & $7(3,2 \%)$ & $19(8,6 \%)$ & $22(10 \%)$ & 1.91 \\
\hline $66(30,1 \%)$ & $56(25,6 \%)$ & $39(17,8 \%)$ & $29(13,2 \%)$ & $29(13,2 \%)$ & 2.54 \\
\hline $63(28,6 \%)$ & $60(27,3 \%)$ & $26(11,8 \%)$ & $42(19,1 \%)$ & $29(13,2 \%)$ & 2.61 \\
\hline $35(15,9 \%)$ & $41(18,6 \%)$ & $17(7,7 \%)$ & $55(25 \%)$ & $72(32,7 \%)$ & 3.4 \\
\hline $56(25,3 \%)$ & $63(28,5 \%)$ & $14(6,3 \%)$ & $41(18,6 \%)$ & $47(21,3 \%)$ & 2.82 \\
\hline $54(24,7 \%)$ & $75(34,2 \%)$ & $30(13,7 \%)$ & $29(13,2 \%)$ & $31(14,2 \%)$ & 2.58 \\
\hline $69(31,4 \%)$ & $67(30,5 \%)$ & $23(10,5 \%)$ & $31(14,1 \%)$ & $30(13,6 \%)$ & 2.48 \\
\hline $102(46,4 \%)$ & $57(25,9 \%)$ & $34(15,5 \%)$ & $9(4,1 \%)$ & $18(8,2)$ & 2.02 \\
\hline $67(30,7 \%)$ & $74(33,9 \%)$ & $28(12,8 \%)$ & $22(10,1 \%)$ & $27(12,4 \%)$ & 2.39 \\
\hline $53(24,7 \%)$ & $66(30,7 \%)$ & $37(17,2 \%)$ & $30(14 \%)$ & $29(13,2 \%)$ & 2.61 \\
\hline $58(26,6 \%)$ & $62(28,4 \%)$ & $42(19,3 \%)$ & $30(14 \%)$ & $26(11,9 \%)$ & 2.56 \\
\hline $72(32,9 \%)$ & $65(29,7 \%)$ & $31(14,2 \%)$ & $21(9,6 \%)$ & $30(13,6 \%)$ & 2.42 \\
\hline $61(27,9 \%)$ & $68(31,1 \%)$ & $28(12,8 \%)$ & $33(15,1 \%)$ & $29(13,2 \%)$ & 2.55 \\
\hline $53(24,7 \%)$ & $72(32,9 \%)$ & $32(14,6 \%)$ & $34(15,5 \%)$ & $28(12,8 \%)$ & 2.6 \\
\hline $107(48,4 \%)$ & $57(25,9 \%)$ & $31(14,2 \%)$ & $11(5 \%)$ & $15(6,8 \%)$ & 1.96 \\
\hline $45(20,6 \%)$ & $66(30,7 \%)$ & $42(19,3 \%)$ & $31(14,1 \%)$ & $34(15,6 \%)$ & 2.74 \\
\hline
\end{tabular}

Table 1: Survey questions and the distribution of the numerical answers. 
therapy or another intervention $[21,22]$ or lifestyle changes such as dietary changes [23,24] or smoking cessation [25].

\section{Applications of multimedia messages}

Multimedia messaging service (MMS) standard allows the user to attach images, video or audio to the message sent by a mobile phone. The images sent via multimedia message can be used in tele-consulting. For instance, a doctor in rural area sends an image of skin condition or x-ray for consultation to a specialist [26]. Multimedia messages containing images have been used as a working consultation tool between, e.g., Plastic surgeons [27], orthopaedics [28] and radiology [29]. Image messages have also been found useful in postoperative management, in which the patient can send an image of surgical wounds to the hospital $[30,31]$. Also video messages have applications in, e.g., Tele-consulting about echocardiography [32]. Audio messaging has been used to communicate short messages for visually impaired receivers [33] (Table 1).

\section{Methodology}

This study was conducted in 2011 in Seinäjoki Central Hospital, which provides secondary care in its region in Western Finland. The region is located $\sim 300 \mathrm{~km}$ from the national capital, Helsinki. The population of the region is $\sim 200,000$. A commercial text messaging solution called bookit [34] is already now in operative use in eight departments of hospital to remind the patients of appointments. However, currently there are about 3-5 persons on each of these departments who are using this solution. In a previous research project, a SMS based application was piloted for psychiatric use in the hospital [18]. The results of piloting program were encouraging, and the department of psychiatry is looking for a commercial text messaging solution for regular use. Also in several other departments of the hospital there is a need for introducing new mobile technology based tools for health care. On the other hand, the goal of the hospital administration is to develop the processes of health care to utilize new mobile tools in a generalized manner. For this purpose, a technology change program involving most of the hospital departments needs to be established. To enable the change, several groups of hospital staff need to be involved, and for this reason we needed survey data on the attitudes and opinions towards mobile phone messaging. We decided to collect this data from all the hospital personnel despite the fact that only minor part of them had previous experience on the use of the mobile messaging tools in their daily work. In this manner we can understand the challenges of introducing these new tools in new areas of health care.

We conducted a survey among the hospital staff, in which we asked their opinions related to different types of mobile phone messaging. Based on the taxonomy presented in the previous section, we prepared a structured questionnaire containing 19 questions. The questions were divided into seven categories according to the taxonomy. The questions in full are presented in table 1, which also presents the results of the survey. The survey contained typical use cases of text messaging and multimedia messaging in health care. The participants were asked to give their opinions on the suitability of these applications on the health care on their field. The question stated in the questionnaire was the following: "Do you find mobile phone text messages or multimedia messaging a feasible way of communication in the following scenarios?". The questions were scored on a five-level scale: yes (1), maybe (2), neutral (3), maybe not (4) and no (5). Each level had a numerical value that is utilized in calculation of statistics based on the answers. For statistical analysis, numerical scores of 1 or 2 were considered positive, 3 indifferent and 4 or 5 negative. In our case we calculated an average value for each question. There was also a field for free text comments in the questionnaire. This field was intended for those who wanted to explain their responses or give additional comments. The survey was carried out as an online web questionnaire using a commercial application, webpropol [35]. The questionnaire was open between june 6th and september 1st, 2011. There was a link to the survey in the hospital intranet. The hospital staff was encouraged to answer the questionnaire by reminder e-mail messages. The total number of participants was 218 of the total target group of 2952 persons, and their distribution is presented in table 2. The survey was targeted for all the medical areas that are present in the hospital, and the answers were received from the most of them. However, the departments of highest response rate were administration ${ }^{1}$ (49 participants), psychiatry (23), rehabilitation (23), internal medicine (14), child and adolescent psychiatry (12), orthopedics (11), gynaecology and obstetrics (10) (Table 2).

\section{Discussion on the Survey Results Comments given by the participants}

There was a free-text comment field in the survey, and 59 of the participants had used it. In general, the comments given by the participants included the following main topics:

- Almost everyone has a mobile phone, and therefore many kinds of routines (like appointments and reminders) in health care can be carried out automatically by mobile phone messaging. This can reduce the workload of the hospital personnel.

- Text message reminders have both benefits and drawbacks: they reduce the number of forgotten and unused appointments, but on the other hand the patient should take the responsibility for remembering the appointments, medication etc., by him or herself.

- Some participants expressed their concerns about the information

\begin{tabular}{|l|c|}
\hline & Participants \\
\hline Specialists & 30 \\
\hline Specializing doctors & 6 \\
\hline Other academic healthcare personnel incl. physicists, chemists, pharmacists, psychologists etc. & 211 \\
\hline Nurses & 53 \\
\hline Other nursing personnel incl. dental nurses, childcare and mental care staff etc. & 150 \\
\hline Assistants incl. laboratory staff, secretaries, research assistants etc. & 43 \\
\hline Maintenance staff incl. Hospital assistants, meal service staff, transportation and technical staff. & 25 \\
\hline Administrative personnel incl. Financial administration, planning, IT services etc. & 350 \\
\hline Total & 354 \\
\hline
\end{tabular}

Table 2: Distribution of the survey participants. 
carried by the text messages: is it understood correctly, is it kept confidential and how one can be sure that the message reaches the correct receiver? For this reason, phone calls and face-to-face discussions were preferred in many cases.

- Some (e.g., Elderly) people do not use the text messaging functionality in their phones, and they use the phones merely for phone calls. For these people, the text messaging based health care services are not an option. On the other hand, younger people use the text messaging on daily basis and many of these application fields are found suitable for them.

\section{Analysis of the numerical answers}

The results of the survey are summarized in table 1. As expected, the applications related to appointments (category a) received the most positive feedback. An exception in this category is question 1 . Based on the free text feedback it seems that the common opinion supports the use of reminding messages, but some participants were concerned whether the messages really reach the correct patient. There was also uncertainty about the ability of all the participants to use text messaging (e.g., Elderly people). However, based on our survey the use of text messages are regarded as useful method for reminding and informing the patients about their appointments. There is also a significant amount of research results that support this, reviewed in [2]. As mentioned before, a text message based system has already adopted for reminding about the appointments in some departments of the hospital. In category $b$, the text message alerts were found to be potential in some cases, but the drawback related to them might be that part of the recipients does not understand the alert correctly. This may cause overreacting and yield to a number of unnecessary phone calls to the hospital. Consequently SMS alerts might work for a limited amount of selected recipient groups, but not for masses.

In category $\mathrm{c}$ we asked about the use of text messages to send the results of the laboratory examination results for the patients. The answers to the question 7 were surprisingly negative. Based on the feedback it seems that the health care personnel are reluctant to send laboratory results to the patients via text message for the following reasons: 1) one cannot be sure that the message reaches the correct person and the confidentiality is guaranteed, 2) the patients do not necessarily understand the results, and therefore it is better that a professional explains them either face-to-face or by phone call. This makes it possible for the patients to get immediate answers to their questions. Also the use of text message in requesting the patient to contact hospital to make appointment for further examinations (question 8) was found to be unsuitable by many participants. This is because these kinds of messages may raise uncertainty and unnecessary concerns among the patients. For this reason it is preferred that the health care personnel contact the patient by phone call to explain the reason for the examinations. Questions in category d (questions 9 and 10) received quite neutral average scores. Based on the comments it seems that sending the results of home measurements or other information is suitable in cases where the patients make the measurements routinely (e.g., Asthma or diabetes), and the messages are automatically managed in the hospital system (i.e. They do not cause any extra work for the hospital staff). Alarm messages (question 11) were regarded as a good idea, but there were some comments that suspected the reliability of the detection of the emergencies by using mobile devices.

The questions related to adherence (category f) received quite neutral opinions. It seems that the participants do not strictly object to these applications, but find some potential problems in transferring the responsibility of remembering appointments, medication etc from the patient to the hospital. On the other hand, the reminders about the medication might suit for some particular groups of patients who have problems with medication adherence. Also the use of text messages to encourage the participants of healthy lifestyle programmes (such as smoking cessation or weight management) was seen mainly as a positive thing.

In the final category, we asked the participants' opinions about the use of multimedia messages in the communication between the hospital and patient. The attitudes towards tele-consultation using multimedia messages containing images (taken by doctors or patients themselves) are mostly positive despite the fact that there is no prior experience on this kind of tele-consultation among the participants. Based on the comments it seems that the main concerns are related to image quality of the mobile phone cameras and the ability of the patients to capture images with adequate quality. Also there was uncertainty about the reliability of the diagnoses that are made merely based on the images. For this reason, e.g., Skin conditions should not be analyzed based on the images, but for instance surgical wounds might be possible to be controlled based on the images. Again, the participants commented that many elderly people cannot probably use the mobile phone camera and image messages. The use of audio messages (question 18) has been considered as a good idea since they can be easily used to send information for the people who have difficulties with vision.

\section{Conclusions}

The results of the survey show that majority of the hospital staff participating in the survey are ready to accept the text messaging systems to at least some area. As was expected, applications related to appointments received the most positive feedback. There are also very valid concerns about e.g., Privacy and confidentiality of the message content. This is obvious since the text message does not provide interactive connection to the receiver, and therefore the sender cannot be sure whether the correct receiver alone is the one that reads the message and whether the message is understood correctly. For this reason, many health care professionals prefer personal one-to-one contact, such as phone call or face-to-face discussion when, e.g., Examination results are communicated to the patients. However, it is apparent that respondents might feel more concerned about security and privacy related to SMS messages than they do in relation to "traditional" contact by phone. After all, there is usually no identification included in person-to-person phone contacts. One potential solution for this problem might be a secure message approach, in which the respondent to the message first receives a notification of a secure message, and responds to this message with his/her personal pin code. After this, the message with confidential content is sent to the respondent.

It should also be noted that introducing new technologies and processes may cause fear of change and reluctance among the hospital staff, and this may also bias the survey results. When introducing new tools and technologies to the hospital environment, we have found that it is essential to ensure efficient information sharing for all the involved people. The key persons from all the relevant departments need also to be participated in the planning of the technology deployment. To facilitate a successful change program, sufficient amount of trainings and information sharing events need to be organized. One good way of make the information sharing more concrete is to provide the personnel with demonstrations, in which they can familiarize with the new tools. In addition, a survey executed among the hospital staff can be a good way of advertising the new concepts to be introduced. In our case, the number of responses indicates that there is interest towards 
Citation: Bäck I, Mäkelä K (2012) Opinions and Attitudes of Health Care Staff towards Mobile Phone Messaging. Primary Health Care 2:125. doi:10.4172/2167-1079.1000125

Page 5 of 5

the text messaging technology, and also those who did not respond, received information about the change in program via e-mail.

There are several reasons for a relatively low response rate. Most important of them was that all the people employed by the hospital were allowed to respond, but the majority of them had no previous connection to the mobile phone messaging in their work. For instance, there were no answers among the maintenance staff, which is natural since they have no prior experience on working with these kinds of applications. In fact, the people who are working with appointments and ways of communications between hospital and patient include some secretaries and a minor part of nurses. According to the free text comments it is possible to conclude that quite many of these people were participating in the survey. On the other hand, in the future there will probably be significantly larger amount of hospital staff involved with the mobile tools, and it is therefore important to get opinions also from those people, even though they have no previous experience on these tools. By allowing all the staff to answer, we got responses from large range of employee groups. However, the responses from different groups had not major differences. From that point of view, it seems that the general opinion scores given by our survey reflects the opinions of the hospital staff relatively well. The results and conclusions of this study are used when the decisions on the future technologies are made in the hospital. It is probable that the text messaging will be taken into wider operative use on several departments, at least in the handling of appointments. It is also very probable that a new survey will be conducted on these departments after the deployment of new technologies, to see how the opinions have been changed.

\section{Acknowledgements}

The authors wish to thank Technology Development Centre of Finland (TEKES) for financial support.

\section{References}

1. Free C, Phillips G, Felix L, Galli L, Patel V, et al. (2010) The effectiveness of M-health technologies for improving health and health services: a systematic review protocol. BMC Res Notes 3: 250.

2. Hasvold PE, Wootton R (2011) Use of telephone and SMS reminders to improve attendance at hospital appointments: a systematic review. J Telemed Telecare 17: 358-364.

3. Wei J, Hollin I, Kachnowski S (2011) A review of the use of mobile phone text messaging in clinical and healthy behaviour interventions. J Telemed Telecare 17: 41-48.

4. Cole-Lewis H, Kershaw T (2010) Text messaging as a tool for behavior change in disease prevention and management. Epidemiol Rev 32: 56-69.

5. Krishna S, Boren SA, Balas EA (2009) Healthcare via cell phones: A systematic review. Telemed J E Health 15: 231-240.

6. Bäck I, Mäkelä K (2012) Mobile phone messaging in health care-where are we now? J Inform Tech Soft Engg 2: 106.

7. Downer SR, Meara JG, Da Costa AC (2005) Use of SMS text messaging to improve outpatient attendance. Med J Aust 183: 366-368.

8. Terry M (2008) Text messaging in healthcare: the elephant knocking at the door. Telemed J E Health 14: 520-524.

9. Shah A (2007) The Future is now- Mobile technology and public health. The Yale Journal of Public Health: 31-34.

10. Cochrane JE, Lowbridge C, Maywood P, Conaty SJ (2009) SMS text messaging for contact follow-up in invasive meningococcal disease. Med J Aust 190: 282 283.

11. Mao Y, Zhang Y, Zhai S (2008) Mobile phone text messaging for pharmaceutical care in a hospital in China. J Telemed Telecare 14: 410-414.

12. Wangberg SC, Årsand E, Andersson N (2006) Diabetes education via mobile text messaging. J Telemed Telecare12: 55-56.

13. Juzang I, Fortune T, Black S, Wright E, Bull S (2011) A pilot programme using mobile phones for HIV prevention. J Telemed Telecare 17: 150-153.
14. Ferrer-Roca O, Cárdenas A, Diaz-Cardama A, Pulido P (2004) Mobile phone text messaging in the management of diabetes. J Telemed Telecare 10: 282285

15. Istepanian RS, Zitouni K, Harry D, Moutosammy N, Sungoor A, et al. (2009) Evaluation of a mobile phone telemonitoring system for glycaemic control in patients with diabetes. J Telemed Telecare 15: 125-128.

16. Prabhakaran L, Chee WY, Chua KC, Abisheganaden J, Wong WM (2010) The use of text messaging to improve asthma control: a pilot study using the mobile phone short messaging service (SMS). J Telemed Telecare 16: 286-290.

17. Holtz B, Whitten P (2009) Managing Asthma with Mobile Phones: A Feasibility Study. Telemed J E Health 15: 907-909.

18. Mäkelä K, Paavola T, Stenman M (2010) Development of Short Message Service Application for Patient-Provider Communication in Clinical Psychiatry. Telemed J E Health 16: 827-829.

19. Jang DW, Sun B, Cho SY, Sohn S, Han KR (2007) Implementation of Ubiquitous Health Care System for Active Measure of Emergencies. Sixth International Conference on Advanced Language Processing and Web Information Technology: 420-425.

20. Sen Gupta G, Mukhopadhyay SC, Sutherland M, Demidenko S (2007) Wireless Sensor Network for Selective Activity Monitoring In a Home for the Elderly. Conference of Instrumentation and Measurement Technology: 1-6.

21. Dunbar PJ, Madigan D, Grohskopf LA, Revere D, Woodward J, et al. (2003) A Two-way Messaging System to Enhance Antiretroviral Adherence. J Am Med Inform Assoc 10: 11-15.

22. Harris LT, Lehavot K, Huh D, Yard S, Andrasik MP, et al. (2010) Two-way text messaging for health behavior change among human immunodeficiency viruspositive individuals. Telemed J E Health 16: 1024-1029.

23. Woolford SJ, Clark SJ, Strecher VJ, Resnicow K (2010) Tailored mobile phone text messages as an adjunct to obesity treatment for adolescents. J Telemed Telecare 16: 458-461.

24. Gerber BS, Stolley MR, Thompson AL, Sharp LK, Fitzgibbon ML (2009) Mobile Phone Text Messaging to Promote Healthy Behaviors and Weight Loss Maintenance: A Feasibility Study. Health Informatics J 15: 17-25.

25. Whittaker R, Maddison R, McRobbie H, Bullen C, Denny S, et al. (2008) A Multimedia Mobile Phone-Based Youth Smoking Cessation Intervention: Findings From Content Development and Piloting Studies. J Med Internet Res 10: e49.

26. Luk R, Zaharia M, Ho M, Levine B, Aoki PM (2009) ICTD for healthcare in Ghana: Two parallel case studies. 3rd International Conference on Information and Communication Technologies and Development (ICTD):118-128.

27. Farber N, Haik J, Liran A, Weissman O, Winkler E (2011) Third generation cellular multimedia teleconsultations in plastic surgery. J Telemed Telecare 17: 199-202.

28. Eranki V, Munt J, Lim MJ, Atkinson R (2010) Consultation of orthopaedics cases using multimedia messaging services. Open Orthop J 4: 164-168.

29. Ng WH, Wang E, Ng I (2007) Multimedia Messaging Service teleradiology in the provision of emergency neurosurgery services. Surg Neurol 67: 338-341.

30. Walker TW, O'Connor N, Byrne S, McCann PJ, Kerin MJ (2011) Electronic follow-up of facial lacerations in the emergency department. J Telemed Telecare 17: 133-136.

31. Martínez-Ramos C, Cerdán MT, López RS (2009) Mobile phone-based telemedicine system for the home follow-up of patients undergoing ambulatory surgery. Telemed J E Health15: 531-537.

32. Lim TH, Choi HJ, Kang BS (2010) Feasibility of dynamic cardiac ultrasound transmission via mobile phone for basic emergency teleconsultation. J Telemed Telecare 16: 281-285.

33. Kummervold PE, Holthe $\mathrm{H}$ (2008) Communicating textual health information to the mobile phones of visually-impaired users. J Telemed Telecare 14: 186-189.

34. http://www.bookit.net/

35. www.webpropol.com 